\title{
Die metatese van alkyne
}

H.C.M. Vosloo

Departement Chemie, Potchefstroomse Universiteit vir Christelike Hoër Onderwys (Vaaldriehoekkampus), Posbus 1174, Vanderbijlpark 1900

J.A.K. du Plessis*

Departement Chemie, Potchefstroomse Universiteit vir Christelike Hoër Onderwys, Potchefstroom 2520

Onwang 23 November 1990); aanvar 28 Jamuarie 1991

\section{UITTREKSEL}

Die alkynmetatesereaksie is 'n direkte gevolg van navorsing oor die bekende en intensief bestudeerde alkeen- of olefienmetatesereaksie. Ongelukkig is hierdie reaksie nie so intensief bestudeer soos die alkeenmetatesereaksie nie, hoofsaaklik weens 'n gebrek aan aktiewe katalisatorsisteme. In die alkynmetatesereaksie word die koolstof-koolstof-trippelbindings gebreek en herrangskik om 'n herverspreiding van alkilidyngroepe te lewer. 'n Oorsig van die reaksie word onder die volgende hoofde gegee: Historiese ontwikkeling en toepassings

Aktiewe katalisatore (beide homogeen en heterogeen)

Kokatalisatore

Alkynsubstrate

Oplosmiddels en

Die verskillende meganismes wat vir alkynmetatese voorgestel is, nl. die paarsgewyse meganisme, die Diels-Alder-meganisme en die metaalkarbynmeganisme.

\section{ABSTRACT}

\section{The metathesis of alkynes}

The alkyne metathesis reaction is a direct result of the known and intensively studied alkene or olefin metathesis reaction. Unfortunately this reaction was never studied as intensively as the alkene metathesis reaction, mainly because of a lack of active catalytic systems. In the alkyn metathesis reaction the carbon-carbon triple bonds are broken and rearranged to give a redistribution of alkylidyne groups. A review of the reaction is given under the following headings:

Historical development and applications

Active catalysts (both homogeneous and heterogeneous)

Cocatalysts

Alkvne substrates

Solvents and

The different mechanisins proposed for alkyne metathesis i.e. the pairwise mechanism, the Diels-Alder mechanism and the metal carbyne mechanism.

\section{HISTORIESE OORSIG EN INLEIDING}

Die termiese metatese van onversadigde organiese verbindings is reeds in 1931 waargeneem toe Schneider en Frolich' propeen by $725^{\circ} \mathrm{C}$ na eteen en buteen omgeskakel het. Die eerste katalitiese metatesereaksie van alkene is egter eers in 1964 deur Banks en Bailey² ontdek toe propeen in die teenwoordigheid van $\mathrm{Mo}(\mathrm{CO})_{6} \cdot \mathrm{Al}_{2} \mathrm{O}_{3}$ by $160^{\circ} \mathrm{C}$ metateseprodukte gelewer het. Hierdie ontdekking het daartoe aanleiding gegee dat 'n groot aantal aktiewe metatesekatalisatorsisteme ontwikkel is, en dat die metatese van alkene indringend ondersoek is. ${ }^{3 .}+$ Rooney en Stewart ${ }^{5}$ het die alkeenmetatesereaksie as 'n transalkilideneringsproses gedefinieer. Dit is 'n proses waarin koolstofkoolstof-dubbelbindings gebreek en herrangskik word om 'n statistiese herverspreiding van alkilideengroepe $\left(R_{1} R_{2} C\right.$ :) te lewer sodat ewewigskonsentrasies van reagense en produkte bereik word. Dit kan soos volg voorgestel word:<smiles>[R1]C([R1])=C([R3])[R3]</smiles><smiles>[R3]C([R1])=C([R3])[R]</smiles>

'n Wye verskeidenheid oorgangsmetaalverbindings kataliseer die metatese van alkene, maar molibdeen, wolfram en renium is die drie oorgangsmetale wat verreweg die aktiefste in klassieke katalisatorsisteme is. ${ }^{3}+$ Die meeste van hierdie katalisatore het egter 'n geskikte Lewissuur as kokatalisator nodig.

In 1968 is die metatese van alkyne die eerste keer deur Pennella et al. ${ }^{6}$ waargeneem. Hulle het die heterogene alkeenmetatesekatalisatorsisteem, $\mathrm{WO}_{3} \cdot \mathrm{SiO}_{2}$, gebruik om die metatese van 2 -pentyn by $350^{\circ} \mathrm{C}$ te laat plaasvind. Die reaksie was egter relatief stadig met alkynoligomeri- 
sasic as 'n newereaksie. In 1974 is die eerste homogene katalisatorsisteem vir alkynmetatese deur Mortreux en Blanchard ${ }^{7}$ ontdek toe $\mathrm{Mo}(\mathrm{CO})_{6}$, 'n oormaat fenolreagens (resorsinol, fenol of $\alpha$-naftol), $p$-tolielfeniclasetileen en dekalien vir 3 ure saam in 'n verseëlde vakuumbuis by $160^{\circ}$ $\mathrm{C}$ verhit is.

Met behulp van ${ }^{13} \mathrm{C}^{-8,9}$ en ${ }^{14} \mathrm{C}$-isotoopmerking ${ }^{10}$ is vasgestel dat die alkynmetatesereaksie die uitruiling van alkilidyngroepe (RC:), met ander woorde 'n transalkilidyneringsproses, behels. Die definisie van Rooney en Stewart, aangepas vir alkyne, geld dus ook vir die alkynmetatesereaksie. Dit kan soos volg voorgestel word:

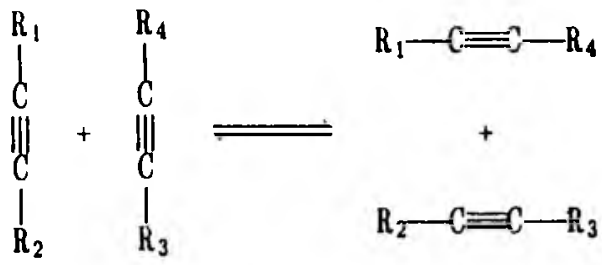

Van die wye verskeidenheid alkyne wat ondersoek is, word produktiewe metatese ${ }^{3}$ egter slegs by digesubstitueerde of interne alkyne waargeneem, terwyl monogesubstitucerde of terminale alkyne hoofsaaklik siklotrimerisasie" "2 of polimerisasie ${ }^{3}$ ondergaan. Die reaksies van alkyne met metatesekatalisatorsisteme kan dus in drie kategorieë ingedeel word:

(I) alkynmetatese

$2 \mathrm{RC} \equiv \mathrm{CR}_{1} \longrightarrow \mathrm{RC} \equiv \mathrm{CR}+\mathrm{R}_{1} \mathrm{C} \equiv \mathrm{CR}_{1}$

(2) alkynsiklotrimerisasie

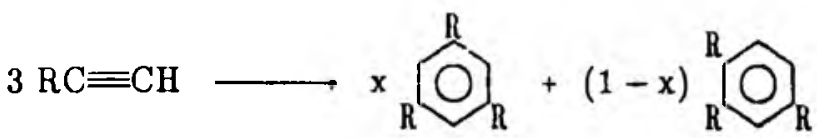

(3) alkynpolimerisasic

$\mathrm{n} \mathrm{RC} \equiv \mathrm{CII} \longrightarrow=[=\mathrm{CR}-\mathrm{CH}=]_{\mathrm{n}}$

Behalwe bogenoemde reaksies word die volgende reaksies ook deur metatesekatalisatore gekataliseer:

(1) leerpolimerisasie van fenielasetileen ${ }^{12}$

$\mathrm{n} \mathrm{PhC} \equiv \mathrm{CH} \longrightarrow$

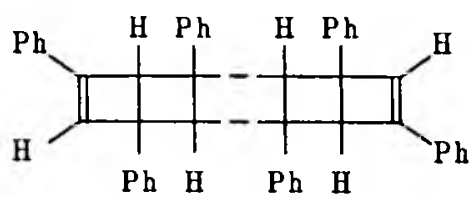

(2) halogenering van alkyne $\mathrm{e}^{13}$

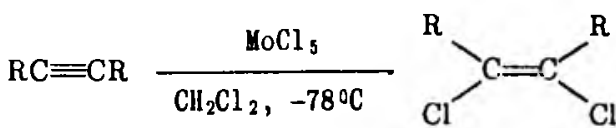

(3) molekulêre herrangskikking't<smiles>C#Cc1ccccc1-c1ccccc1/C=C/C</smiles><smiles>CC(C)(C)C(C)(C)c1ccccc1</smiles><smiles>C/C=C\c1cc2ccccc2c2ccccc12</smiles>

(4) fotochemiese reaksies van siklo-oktyn ${ }^{15}$

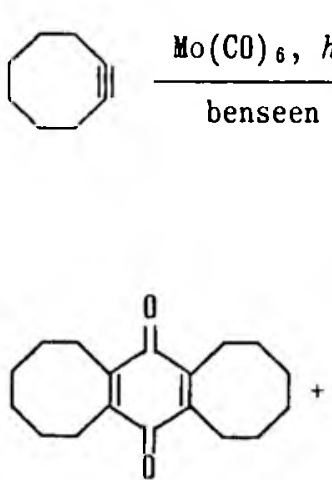

$1 \%$

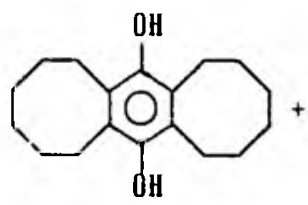

$13 \%$

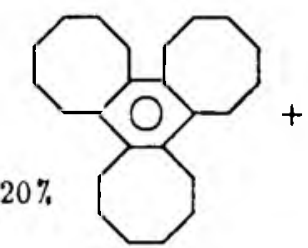

$\left(\mathrm{C}_{8} \mathrm{H}_{12}\right)_{3} \mathrm{Mo}(\mathrm{CO})_{3}$ $3 \%$

(5) Hoëtemperatuur $\left(>100^{\circ} \mathrm{C}\right.$ ) Friedel-Craftsasilering en -alkilering van aromate met $\mathrm{Mo}(\mathrm{CO})_{6}$ of (tolueen) $\mathrm{Mo}(\mathrm{CO})_{3}$-haloalkaanverbindingmengsels. ${ }^{\text {th }}$

Die bestudering van die alkynmetatesereaksie is nog nie naastenby so omvattend en deeglik gedoen soos in die geval van alkeenmetatese nie, sodat min toepassings van alkynmetatese bekend is. 'n Betekenisvolle toepassing is die sintese van stereospesifieke feromone soos byvoorbeeld $\omega$ fluoro-oleïensuur ${ }^{17}$,'n komponent van die vrug van die Dichapetalum toxicarium-struik wat as rottegif gebruik word. $\omega$-Fluoro-oleïensuur kan berei word deur die kruismetatese van gefunksionaliseerde alkyne ${ }^{18}$ gevolg deur selektiewe hidrogenering ${ }^{19}$ van die metateseproduk:

$$
\begin{aligned}
& \mathrm{F}\left(\mathrm{CH}_{2}\right)_{8} \mathrm{C} \equiv \mathrm{CR}+\mathrm{RC} \equiv \mathrm{C}\left(\mathrm{CH}_{2}\right)_{7} \mathrm{COOH} \\
& \mathrm{F}\left(\mathrm{CH}_{2}\right)_{8} \mathrm{C} \equiv \mathrm{C}\left(\mathrm{CH}_{2}\right)_{7} \mathrm{COOH}
\end{aligned}
$$

$$
\begin{aligned}
& \mathrm{F}\left(\mathrm{CH}_{2}\right)_{8} \mathrm{C} \equiv \mathrm{C}\left(\mathrm{CH}_{2}\right)_{7} \mathrm{COOH}+\mathrm{H}_{2} \stackrel{\text { Lindlar }}{\longrightarrow} \\
& \mathrm{F}\left(\mathrm{CH}_{2}\right)_{8} \mathrm{CH}=\mathrm{CH}\left(\mathrm{CH}_{2}\right)_{7} \mathrm{COOH}
\end{aligned}
$$

As gevolg van die afwesigheid van aktiwiteit ten opsigte van gefunksionaliseerde alkene en selektiwiteit ten opsigte van E/Z-isomerie kan alkeenmetatese nie vir hierdie sintese gebruik word nie. ${ }^{20}$

Verdere toepassings van alkynmetatese kom in dic polimerisasieveld voor, maar dit is onduidelik of die meganisme wat hier ter sprake is, dié van metatese is. ${ }^{21}$ 


\section{KATALISATORE}

Metatesekatalisatore kan in drie hoofklasse verdeel word:22

(1) Ziegler-Natta-tipe sisteme

(2) ondersteunde oorgangsmetaaloksiedes en -karboniele

(3) ander oorgangsmetaalverbindings

Alkynmetatese word egter slegs deur katalisatore uit klasse (2) en (3) gekataliseer en dan hoofsaaklik katalisatore gebaseer op die Groep VI-oorgangsmetale molibdeen en wolfram. Groep VI-oorgangsmetaalverbindings wat die metatese van alkyne kataliseer, kan verder in twee subklasse verdeel word:

(1) metaalkarboniele en derivate

(2) metaalkarbyne

Net soos in die geval van alkeenmetatese is aanvanklik van heterogene katalisatorsisteme gebruik gemaak om die alkynmetatesereaksies te kataliseer en het 'n klemverskuiwing plaasgevind met die ontdekking van die eerste homogene sisteem. Heelwat meer homogene as heterogene sisteme is daarom dus bekend. ${ }^{3}$ Terwyl wolframverbindings die volopste en waarskynlik belangrikste groep alkeenmetatesekatalisatore is, is molibdeenverbindings waarskynlik die belangrikste vir alkynmetatese. Molibdeen- en wolframverbindings is egter nie die enigste aktiewe alkynmetatesekatalisatore nie, enkele reniumkatalisatore is ook bekend. ${ }^{23}$

\subsection{Heterogene katalisatorsisteme}

Die heterogene $\mathrm{WO}_{3} . \mathrm{SiO}_{2}$-sisteem ${ }^{6}$ is die eerste alkynmetatesekatalisator wat in 'n vastebedreaktor by $350^{\circ} \mathrm{C}$ 2-pentyn na 2-butyn en 3-heksyn omgeskakel het. Die omskakeling het met $53 \%$ selektiwiteit plaasgevind. Geen metatese is waargeneem indien slegs $\mathrm{SiO}_{2}$ gebruik is nie. Die $\mathrm{MoO}_{3} \cdot \mathrm{SiO}_{2}$-sisteem is ook ondersoek. ${ }^{10}$ 2-Heksyn het $40 \%$ metateseprodukte gelewer, terwyl propyn slegs $90 \%$ siklotrimerisasie ondergaan het. Propyn inhibeer ook die metatesereaksie van 2-heksyn indien dit by die reaksiemengsel gevoeg word. Daar is ook gevind dat die $\mathrm{Mo}(\mathrm{CO})_{6} . \mathrm{SiO}_{2}$-sisteem, ${ }^{9}$ " 11 wat voorheen vir alkeenmetatese gebruik is, die metatese van 2-heksyn by $220^{\circ} \mathrm{C}$ kataliseer.

Mortreux et al. ${ }^{9}$ het gepoog om die aktiwiteit en selektiwiteit van die $\mathrm{MoO}_{3} . \mathrm{SiO}_{2}$-sisteem te verhoog deur die bereidingsmetodes te verbeter. Daar is gevind dat die aktiwiteit tot $52 \%$ en die selektiwiteit tot $73 \%$ toeneem indien die $\mathrm{MoO}_{3}$-inhoud na 30\% verhoog word. Die selektiwiteit van die $\mathrm{Mo}(\mathrm{CO})_{6} . \mathrm{SiO}_{2}$-sisteem is ook met voorafbehandeling verhoog. ${ }^{9}$ Met behulp van infrarooispektroskopie $^{24}$ is aangetoon dat die karbonielgroepe tydens die voorafbehandeling verdwyn en dat die aktiewe katalisator 'n molibdeenioon in 'n hoër oksidasietoestand as die nultoestand is. $\mathrm{Na}$ aanleiding hiervan is ' $\mathrm{n}$ hipotese gepostuleer ${ }^{9}$ dat maksimum aktiwiteit verkry kan word deur ' $n$ inpregnasiemetode te gebruik wat die afsetting van 'n uniforme monolaag Mo-atome toelaat. Hierdie monolaag is langs $\mathrm{Si}-\mathrm{O}-\mathrm{Mo}$-bindings aan die ondersteunersmateriaal gebind. Die hipotese is bevestig deur die bereiding van 'n koördinasiekatalisator wat na oksidasie monolaag spe- sies lewer:25

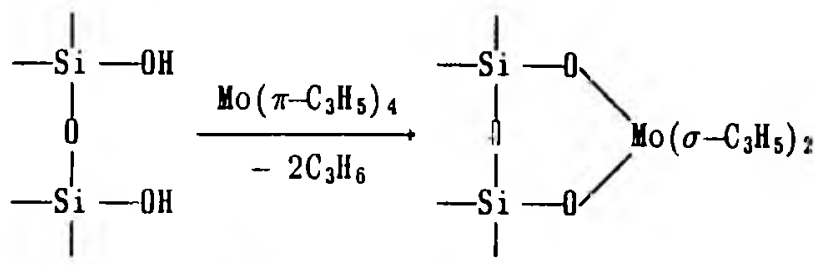<smiles>C[Si]1(C)O[Si](C)(C)O[As](=O)(=O)O1</smiles>

$\left[\mathrm{HOO}_{3} \cdot \mathrm{SiO}_{2}\right]^{*}$

Die geoksideerde produk is vir $\mathrm{MoO}_{3}-\mathrm{SiO}_{2}-$ en $\mathrm{MoO}_{3}-\mathrm{Al}_{2} \mathrm{O}_{3}$-sisteme gepostuleer. ${ }^{26}$ Die volgende verhoogde selektiwiteite is waargeneem:

$$
\begin{array}{lr}
\text { geïmpregneerde- } \mathrm{MoO}_{3} \cdot \mathrm{SiO}_{2} & 75-80 \% \\
\mathrm{Mo}\left(\alpha-\mathrm{C}_{3} \mathrm{H}_{5}\right) \cdot \mathrm{SiO}_{2} & 85-90 \% \\
{\left[\mathrm{MoO}_{3} \cdot \mathrm{SiO}_{2}\right]^{*}} & 95-100 \%
\end{array}
$$

Die aktiewe katalisator blyk dus die monolaagspesie, $\left[\mathrm{MoO}_{3} \cdot \mathrm{SiO}_{2}\right]^{*}$, te wees wat van enige ongewenste aktiewe posisies, veral in dié wat tot siklotrimerisasie aanleiding gee, vry is en by lae Mo-bevattende katalisatore voorkom, 9,10

Ten slotte, 'n $\mathrm{CoO}-\mathrm{MoO}_{3} \cdot \mathrm{SiO}_{2}$-sisteem is voorgestel wat die metatesereaksie van terminale alkyne kataliseer. ${ }^{5}$ Fenielasetileen het by 'n temperatuur van $400^{\circ} \mathrm{C}$ en 'n ruimtesnelheid van $6 \mathrm{~h}^{-1} \mathrm{MoO}_{3} \cdot \mathrm{SiO}_{2}$ wat $1 \% \mathrm{CoO}$ bevat $30-40 \%$ difenielasetileen gelewer. Met verlaging van die ruimtesnelheid is slegs polimerisasie waargeneem, terwyl 'n verhoging in die ruimtesnelheid en temperatuur $\left(500-550^{\circ} \mathrm{C}\right) 60-70 \%$ defenielasetileen tot gevolg gehad het.

2.2 Metaalkarboniel- en -afgeleide katalisatorsisteme Die eerste en waarskynlik bekendste katalisator vir alkynmetatese is $\mathrm{Mo}(\mathrm{CO})_{6}{ }^{3,5,7,21} \mathrm{Mo}(\mathrm{CO})_{6}$ kataliseer slegs die metatese van interne alkyne en dan alleenlik indien 'n oormaat van 'n fenoliese kokatalisator teenwoordig is. Temperature waarby hierdie sisteme effektief is, is heelwat laer as dié van heterogene sisteme, naamlik $110-160^{\circ}$ $\mathrm{C}$ in plaas van $350^{\circ} \mathrm{C}$. ${ }^{3,}$ Tipiese resultate is $55 \%$ aktiwiteit en $88 \%$ selektiwiteit.

Verskeie $\mathrm{Mo}(\mathrm{CO})_{6}$-afgeleide katalisatore is ook ondersoek:

$\left(\mathrm{Ph}_{3} \mathrm{P}\right) \mathrm{Mo}(\mathrm{CO})_{5},{ }^{27}\left[\mathrm{Bu}_{4} \mathrm{~N}\right]^{+}\left[\mathrm{Mo}(\mathrm{CO})_{5}\right]^{-, 27}$ trans $-\left[\mathrm{Mo}(\mathrm{CO})_{4}\left(\mathrm{PPh}_{3}\right)_{2}\right],{ }^{28}$ (norbornadieen) $\mathrm{Mo}(\mathrm{CO})_{4},{ }^{27}$

$\mathrm{Mo}(\mathrm{CO})_{3}\left(\mathrm{C}_{6} \mathrm{H}_{5} \mathrm{OH}\right),{ }^{28,}{ }^{29} \mathrm{Mo}(\mathrm{CO})_{3}\left(\mathrm{NH}_{3}\right)_{3},{ }^{28}$

$\mathrm{Mo}(\mathrm{CO})_{3}(\mathrm{MeCN})_{3},{ }^{28}$

$(1,3,5$-sikloheptatriëniel $) \mathrm{Mo}(\mathrm{CO})_{3},{ }^{27}$

cis-[Mo(CO) $\left.)_{2}\left(\mathrm{Ph}_{2} \mathrm{PCH}_{2} \mathrm{PPh}_{2}\right)_{2}\right],{ }^{28}$

(1, 3-sikloheksadiëniel $)_{2} \mathrm{Mo}(\mathrm{CO})_{2}, 7$

$\mathrm{Mo}(\mathrm{acac})_{3},{ }^{27} \mathrm{MoO}_{2}(\mathrm{acac})_{2},{ }^{18,}{ }^{77} \mathrm{OMo}\left(\mathrm{OC}_{6} \mathrm{H}_{5}\right)_{4},{ }^{27}$ en

$\mathrm{MO}(\mathrm{NO})_{2} \mathrm{Cl}_{2}(\mathrm{py})_{2} \cdot{ }^{27}$ 
Die metateseaktiwiteit van bogenoemde katalisatore wissel van uitstekend (relatief tot $\mathrm{MO}(\mathrm{CO})_{6}$ ) tot baic swak. $\mathrm{Mo}(\mathrm{CO})_{3} \mathrm{~L}_{3}$-katalisatore $\left(\mathrm{L}=\mathrm{NH}_{3}\right.$ of $\left.\mathrm{MeCN}\right)$ benodig geen fenoliese kokatalisator nie. ${ }^{29}$

$\mathrm{W}(\mathrm{CO})_{6}-\mathrm{C}_{6} \mathrm{H}_{5} \mathrm{OH}-\mathrm{C}_{6} \mathrm{H}_{5} \mathrm{CH}_{3}$ kataliseer nie die alkynmetatesereaksic nie, ${ }^{24}$ terwyl slegs van een $\mathrm{W}(\mathrm{CO})_{6}$-derivaat, naamlik $\mathrm{W}(\mathrm{CO})_{4} \mathrm{Cl}_{2}$, melding gemaak word, wat 'n swak katalisator vir alkynmetatese is. ${ }^{n}$

\subsection{Metaalkarbynkatalisatorsisteme}

'n Belangrike ontwikkeling op die gebied van metatesekatalisatore was die ontdekking van metaalkarbyn- of alkilidynkomplekse. Daar word tussen twee tipes onderskei:

(1) Fischer-tipe, ${ }^{30} \mathrm{X}(\mathrm{CO})_{4} \mathrm{M} \equiv \mathrm{CR}$ met $\mathrm{X}=\mathrm{Cl}, \mathrm{Br}$ en $\mathrm{M}=\mathrm{W}, \mathrm{Mo}, \mathrm{R}=$ alkiel- of arielgroep; en

(2) Schrock-tipe, ${ }^{3 !}(\mathrm{RO})_{3} \mathrm{M} \equiv \mathrm{CBu}^{1}, \mathrm{R}=$ alkiel- of arielgroep en $\mathrm{M}=\mathrm{W}$, $\mathrm{Mo}$.

Hierdie ontdekkings was belangrik, veral in die lig daarvan dat 'n metaalkarbynmeganisme vir alkynmetatese voorgestel is. ${ }^{32}$ Die Fischer-tipe metaalkarbyne is egter nie aktiewe metatesekatalisatore nie. ${ }^{33}$ Sommige van die Schrock-tipe metaalkarbyne is egter uiters aktiewe katalisatore vir die metatese van interne alkyne (byvoorbeeld wanneer $\mathrm{M}=\mathrm{W}$ en $\mathrm{OR}=\mathrm{OBu} \mathbf{u}^{\prime}$ ), ${ }^{31}$ terwyl ander (byvoorbeeld $\left.\mathrm{W}\left(\mathrm{CBU}^{\prime}\right)\left(\mathrm{O}-2,6-\mathrm{Pr}_{2}{ }_{2} \mathrm{C}_{6} \mathrm{H}_{3}\right)_{3}\right)$ met interne alkyne reageer om metallasiklobutadieenkomplekse van die tipe $\mathrm{M}\left(\mathrm{C}_{3} \mathrm{R}_{3}\right)(\mathrm{OR})_{3}$ te lewer. Laasgenoemde verbindings is egter swak katalisatore as gevolg van 'n stadige tempobeperkende verlies van alkyne vanuit die $W_{3}$-ring om $\mathrm{W}\left(\mathrm{CR}^{\prime}\right)(\mathrm{OR})_{3}$-komplekse te lewer. ${ }^{34}$

Die volgende Schrock-tipe metaalkarbynkomplekse is aktiewe metatesekatalisatore:

$(\mathrm{RO})_{3} \mathrm{~W} \equiv \mathrm{CBu}^{\prime},{ }^{13}\left(\mathrm{R}=\mathrm{Bu}^{\prime}, 2,6-\mathrm{Pr}_{2} \mathrm{C}_{6} \mathrm{H}_{5}, \mathrm{CH}\left(\mathrm{CF}_{3}\right)_{2}\right.$, $\left.\mathrm{CCH}_{3}\left(\mathrm{CF}_{3}\right)_{2}\right) ; \mathrm{Cl}_{3}\left(\mathrm{Et}_{3} \mathrm{PO}\right) \mathrm{W} \equiv \mathrm{CBu}^{\prime} ; 35$

$\mathrm{Cl}_{3}\left(\mathrm{Me}_{3} \mathrm{P}\right)_{3} \mathrm{~W} \equiv \mathrm{CBu}^{\mathrm{i}} ;^{35}\left[\mathrm{Et}_{4} \mathrm{~N}\right]^{+}\left[\mathrm{Cl}_{4} \mathrm{~W} \equiv \mathrm{CBu}^{\mathrm{i}}\right]^{35}$

en $\left(\mathrm{Pr}_{2}{ }_{2} \mathrm{~N}\right)_{3} \mathrm{~W} \equiv \mathrm{CBu}^{\prime}{ }^{36}$

Die gebruik van hierdie wolframkarbynkomplekse hou besliste voordele as katalisatore vir die alkynmetatesereaksie in:

(1) Geen fenoliese kokatalisator is nodig nie.

(2) Die meeste metatesereaksies vind by kamertemperatuur plaas.

(3) Hoë metateseaktiwiteit (word toegeskryf aan die hoë oksidasietoestand $(+6)$ waarin wolfram verkeer). ${ }^{31 \mathrm{c}}$

(4) Ewewig word vinnig bereik. Veral die $10 \mathrm{~min}$. vir die $\left(\mathrm{Bu}^{\prime} \mathrm{O}\right)_{3} \mathrm{~W} \equiv \mathrm{CBu}^{\prime} / \mathrm{C}_{6} \mathrm{H}_{5} \mathrm{Me} / 3$-heksyn-sisteem is indrukwekkend. ${ }^{35}$
Die tempo van die metatesereaksies wat deur metaalkarbyne gekataliseer word, hang veral van twee veranderlikes af: ${ }^{31 \mathrm{~b}}$

(1) Keuse van die oorgangsmetaal.

Die tempo van die metatesereaksie neem drastics af met molibdeenkarbyne. ${ }^{n}$

(2) Keuse van die alkoksiedligand.

Groot alkoksiedligande is in alle gevalle nodig om die deaktivering van die oorgangsmetale deur oorbruggende alkoksiedligande te voorkom. ${ }^{3}$ Een geval is ook bekend waar die verandering van dic alkoksiedligand ' $n$ verandering in die meganisme, van 'n tempobeperkende dissosiasie van die alkyn vanaf die metallasikliese verbinding na 'n assosiatiewe reaksie tussen die metallasikliese verbinding en die alkyn, teweeggebring het. ${ }^{38}$

'n Verskeidenheid molibdeenkarbynkomplekse van die Schrock-tipe is berci in 'n poging om te bepaal of Mo(VI) alkilidynkomplekse die aktiewe spesies in die molibdeensisteme is. ${ }^{39}$ Aanvanklik is molibdeenkarbyne soos die aktiewe wolframkarbyn, $(\mathrm{RO})_{3} \mathrm{~W} \equiv \mathrm{CBu}^{1}$, berei en vir alkynmetateseaktiwiteit getoets: ${ }^{40}$ $\left(\mathrm{Bu}^{\prime} \mathrm{O}\right)_{3} \mathrm{Mo} \equiv \mathrm{CBu}^{\prime}$ het geen metateseaktiwiteit vertoon nie, terwyl $\left(\mathrm{Pr}^{\prime} \mathrm{O}\right)_{3} \mathrm{Mo} \equiv \mathrm{CBu}^{\prime}$ en $\left(\mathrm{Me}_{3} \mathrm{CCH}_{2} \mathrm{O}\right)_{3}$ $\mathrm{Mo} \equiv \mathrm{CBu}^{\prime}$ polimere gevorm het. Laasgenoemde twee het wel aanvanklik metateseprodukte opgelewer wat later moontlik deur die polimere verbruik is. ${ }^{40}$ Die swak metateseaktiwiteit van bogenoemde sisteme is soos volg verklaar: ${ }^{39}, 40$

(1) Groot alkoksiedgroepe is nodig om polimerisasie te voorkom en

(2) $\mathrm{Mo}(\mathrm{VI})$ is baie minder elektrofilies as W(VI).

Molibdeenkarbyne is toe berei waarin bogenoemde eienskappe uitgeskakel is deur van groot elektrontrekkende alkoksiedligande gebruik te maak: ${ }^{+1}$

$$
\begin{aligned}
& {\left[\left(\mathrm{CF}_{3}\right) \mathrm{CMe}_{2} \mathrm{O}\right]_{3} \mathrm{Mo} \equiv \mathrm{CBu}^{\prime}} \\
& {\left[\left(\mathrm{CF}_{3}\right)_{2} \mathrm{CMeO}\right)_{3} \mathrm{Mo} \equiv \mathrm{CBu}^{\prime}} \\
& \text { mer-(dme })\left(\left(\mathrm{CF}_{3}\right)_{3} \mathrm{CO}\right)_{3} \mathrm{Mo} \equiv \mathrm{CBu}^{\prime} \\
& \text { mer-(dme })\left[\left(\mathrm{CF}_{3}\right)_{2} \mathrm{CMeO}\right)_{3} \mathrm{Mo} \equiv \mathrm{CBu}^{\prime} \\
& \text { mer-(dme })\left[\left(\mathrm{CF}_{3}\right)_{2} \mathrm{CHO}\right)_{3} \mathrm{Mo} \equiv \mathrm{CBu}^{\prime} \\
& {\left[2,6-\mathrm{Pr}^{\prime} \mathrm{C}_{6} \mathrm{H}_{3} \mathrm{O}\right)_{3} \mathrm{Mo} \equiv \mathrm{CBu}^{\prime}}
\end{aligned}
$$

Al hierdie molibdeenkarbynkomplekse kataliseer die metatese van alkyne teen omsettingsnelhede wat wissel van minder as $1 \mathrm{~min}$. tot $30 \mathrm{~min}$.

Ten spyte daarvan dat reniumkatalisatore bekende alkeenmetatesekatalisatore is, is hulle onbekend in alkynmetatese. 'n Renium(VII)monoimido-bis-alkoksineopentilidynkompleks is egter berei wat by $25^{\circ} \mathrm{C}$ vinnig met verskeie interne alkyne reageer om metateseprodukte en 'n renasiklobutadieenkompleks te lewer: ${ }^{23}$

$$
\begin{aligned}
& \|_{(\mathrm{RO})}{ }_{2} \mathrm{Re} \equiv \mathrm{CBu}^{\mathrm{t}}+\mathrm{R}_{1} \mathrm{C}=\mathrm{CR}_{1}= \\
& \mathrm{R}=\mathrm{CMe}\left(\mathrm{CF}_{3}\right)_{2} \\
& \mathrm{Ar}=2,6-\mathrm{Pr}_{2} \mathrm{C}_{6} \mathrm{H}_{3}
\end{aligned}
$$

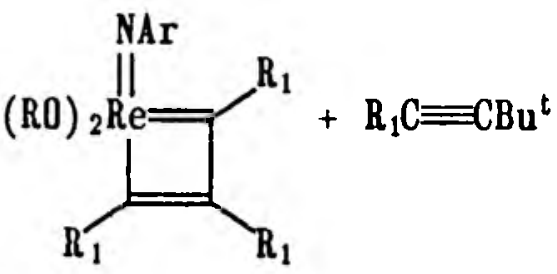




\section{KOKATALISATORE}

Die meeste alkynmetatesekatalisatore het 'n oormaat fenoliese kokatalisator nodig om aktief vir alkynmetatesc te wees. Verskeie fenoliese verbindings word suksesvol gebruik: ${ }^{\text {, } 21}$

$\mathrm{C}_{6} \mathrm{H}_{5} \mathrm{OH}, \mathrm{Cl}^{m} \mathrm{C}_{6} \mathrm{H}_{4} \mathrm{OH}, \mathrm{Br}^{\mathrm{P}} \mathrm{C}_{6} \mathrm{H}_{4} \mathrm{OH}$, resorsinol en $\alpha$ naftol.

Sekere fenoliese verbindings, kinol en katesjol, onderdruk alkynmetatese moontlik deur die vorming van kinone: ${ }^{28}$<smiles></smiles>

Verskeic sisteme is aktief in die afwesigheid van 'n fenoliese kokatalisator:

$\operatorname{Mo}(\mathrm{CO})_{3} \mathrm{~L}_{3}\left(\mathrm{~L}=\mathrm{NH}_{3}, \mathrm{MeCN}\right),{ }^{28}$ metaalkarbyne $\mathrm{e}^{23,}, 30-40$

Fenolverbindings aktiveer slegs alkynmetatese en nie alkeenmetatese nie. ${ }^{3 .}{ }^{41}$ Lewissure is nodig om aktiewe alkeenmetatesekatalisatore te lcwer. Enkele katalisatore vir alkynmetatese het egter bo en behalwe 'n fenol ook 'n Lewissuur nodig: ${ }^{27}$

$\mathrm{MoO}_{2}(\mathrm{acac})_{2}-\mathrm{Et}_{3} \mathrm{Al}-\mathrm{C}_{6} \mathrm{H}_{5} \mathrm{OH}$ en $\mathrm{MoO}(\mathrm{OPh})_{4}-\mathrm{Et}_{3} \mathrm{Al}-\mathrm{C}_{6} \mathrm{H}_{5} \mathrm{OH}$

Slegs 'n beperkte aantal Lewissure gee effektiewe mengsels:

$\mathrm{AlMe}_{3}, \mathrm{AlBu}_{3}, \mathrm{HAlBu}_{2}, \mathrm{AlEt}_{3}$, terwyl Et $\mathrm{AlCl}_{2}, \mathrm{EtAlCl}{ }_{2}$, $\mathrm{ZnEt}_{2}, \mathrm{SnMe}_{4}, \mathrm{NaBH}_{4}$ en $\mathrm{LiAlH}_{4}$ totaal onaktief is. ${ }^{27}$

\section{ALKYNSUBSTRATE}

Slegs interne alkyne, op enkele uitsonderings na, ondergaan metatese. ${ }^{3,2}$ 'n Groot verskeidenheid interne alkyne ondergaan metatese: ${ }^{3}$

2-pentyn, ${ }^{6,41}$ 2-heksyn, ${ }^{9,10} 3$-heptyn, , ${ }^{2.35,42,44} 4$-nonyn, 27.42 .43 1-feniel-l-butyn, 9. 35. 42.44 1-feniel-l-pentyn, ${ }^{8}$ p-toliel fenielasetileen, ${ }^{7.28,36,42.43}$ en 3-heksyn en difenielasetileen. ${ }^{35.44}$

In teenstelling met die swak aktiwiteit en selektiwiteit van gefunksionaliseerde alkene ten opsigte van metatese ondergaan gefunksionaliseerde alkyne geredelik metatese. ${ }^{18}$ Die metatese van gefunksionaliseerde alkyne hou verskeie voordele in: ${ }^{18}$

(1) hoë selektiwiteit en aktiwiteit;

(2) geen isomerisasie van substrate en produkte kom voor nie; en

(3) Die trippelbinding kan met behulp van selektiewe hidrogenering ${ }^{19}$ verwyder word om spesifiek E- of Z-alkene te lewer.

Verskeie gefunksionaliseerde alkyne het metatese sukses vol ondergaan:

$\mathrm{Cl}\left(\mathrm{CH}_{2}\right)_{3} \mathrm{C} \equiv \mathrm{CEt},{ }^{18} \mathrm{CH}_{2}=\mathrm{CHCH}_{2} \mathrm{C} \equiv \mathrm{CBu}$ (slegs die trippelbinding het metatese ondergaan $)^{18}$ en $\mathrm{C}_{6} \mathrm{H}_{5} \mathrm{C} \equiv \mathrm{C}\left(\mathrm{CH}_{2}\right)_{2} \mathrm{X}\left(\mathrm{X}=\mathrm{OH}, \mathrm{OCOCH}_{3}\right.$, $\left.\mathrm{Br}, \mathrm{COOCH}_{3}, \mathrm{CN}\right) .^{29}$

Siklotrimerisasie is ook by interne alkyne waargeneem. ${ }^{45}$ Terminale alkyne gee uitsluitlik siklotrimerisasie ${ }^{3,10,} 11$ en polimerisasie ${ }^{2}$. Die $\mathrm{CoO}-\mathrm{MoO}_{3} \cdot \mathrm{SiO}_{2}$-sisteem ${ }^{5}$ het egter metatese met 1-pentyn en 3-meticlbut-3-een-1-yn (beide die bindings het metatese ondergaan) gegec.

Siklo-alkyne ondergaan net soos die siklo-alkene ringopeningpolimerisasie. ${ }^{46,47}$ Die oligomerisasie van siklodekyn om ' $n$ reeks oligomere, $\left(\mathrm{C}_{10} \mathrm{H}_{16}\right)_{n} \mathrm{n}=\mathrm{I}-6$, te gee is 'n goeie voorbeeld. ${ }^{4 \times}$

\section{OPLOSMIDDELS}

'n Verskeidenheid oplosmiddels word in metatesereaksic gebruik, ${ }^{3}$ waarvan n-heptaan, n-oktaan, tolueen en dekalien die meeste gebruik word. Geen studic is egter gepubliseer wat melding van die invloed van die oplosmiddel op die alkynmetatesereaksie maak nie. Die betrokkenheid van oktaan en tolueen is egter getoon in 'n voorgestelde meganisme vir die alkynmetatesereaksie. ${ }^{28}$

\section{MEGANISME}

Drie meganismes is vir die alkynmetatesereaksie voorgestel. 3. 21. 49

(1) Paarsgewyse meganisme langs 'n metallosiblobutadieentussenverbinding ${ }^{10}$

Die metallosiklobutadieenmeganisme is voorgestel na aanleiding van 'n meganisme wat vir die siklotrimerisasie van alkyne ondersoek is: ${ }^{50}$

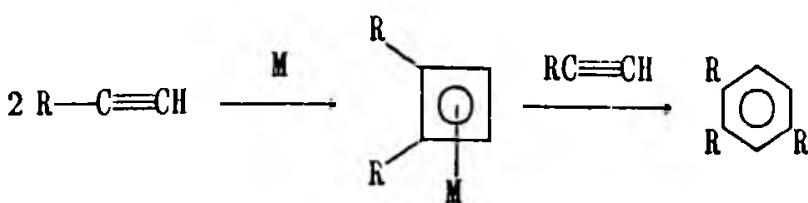

Die metallosiklobutadieenmeganisme sien soos volg daaruit:

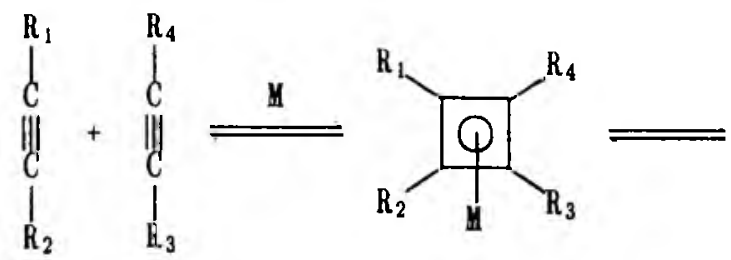

$\mathrm{R}_{1}-\mathrm{C} \equiv \mathrm{C}-\mathrm{R}_{4}$

$+$

$\mathrm{R}_{2}-\mathrm{C} \equiv-\mathrm{R}_{3}$

Hierdie meganisme is egter as onwaarskynlik vir alkynmetatese beskou omdat kobaltosiklobutadieenkomplekse "ongewoon stabiel" is en die gekondenseerde fase termiese ontbinding van hierdie komplekse nie alkynprodukte lewer nie. ${ }^{32.51}$ Fritch en Volhardt ${ }^{52}$ ondersteun egter hierdie meganisme nadat flits- 
vakuumpirolise van $\eta^{4}$-tetrafenielsiklobutadieen$\eta^{5}$-siklopentadiënielkobalt alkyne lewer:
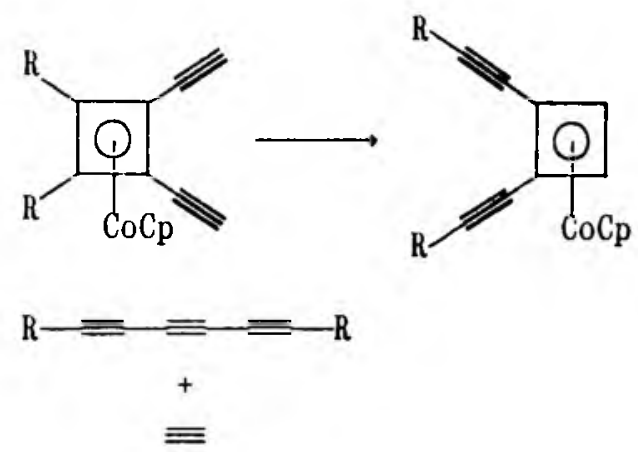

(2) Diels-Alder-reaksie langs 'n metallasiklopentadieentussenverbinding?2

Hierdie meganisme behels die aanvanklike koördinering van twee alkynmolekules aan die katalisator om in metallasiklopentadieenverbinding te lewer wat 'n Diels-Alder-reaksic met 'n derde alkynmolekuul ondergaan. 'n Metallanorbornadieen vorm as tussenverbinding wat dan op sy beurt ontbind om metatese, ontaarde metatese en siklotrimerisasie te gee.

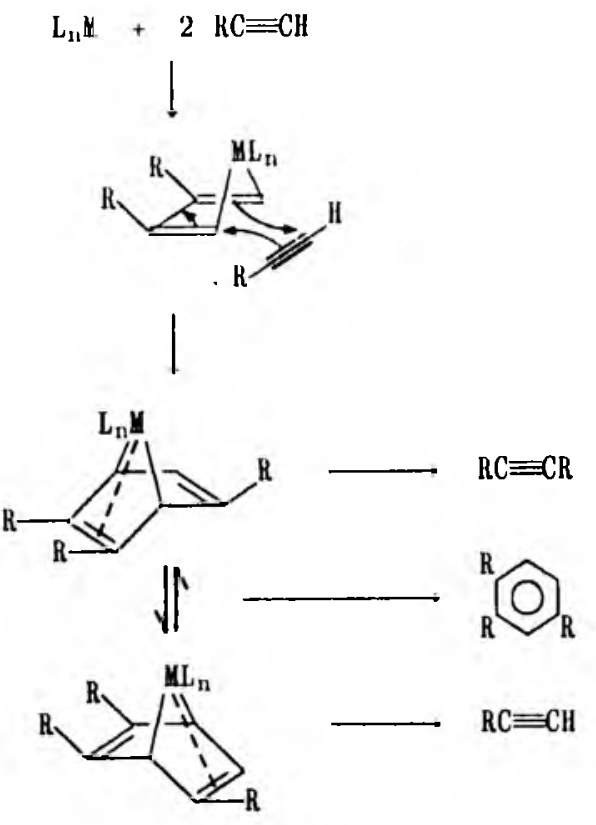

Hierdie meganisme is in ooreenstemming met die cksperimenteel goed ondersteunde voorgestelde aromatiseringsmeganisme van difenielasetilene deur Groep V-metaalkarboniele. ${ }^{53}$ Alkynmetatese word in hierdie meganisme as 'n newereaksie van die siklotrimerisasiercaksie beskou en nie as 'n transalkilidyneringsproses nie. Die meganisme word verder deur die volgende waarnemings ondersteun: ${ }^{22}$

(a) die vorming van die metateseprodukte is nie katalities nie,

(b) die statistiese 1:2:1-verhouding van die drie moontlike alkyne is nie waargeneem nie en

(c) die algemeen aanvaarde alkeenmetatesemeganisme het die koördinasie nodig van twee substraatmolekules en nie drie nie.

Hierdie meganisme ontvang egter min steun as ' $\mathrm{n}$ algemene meganisme vir die metatese van alkyne. ${ }^{3}$
(3) Metalkarbynmeganisme langs 'in metallasiklobutadieentussenverbinding ${ }^{32}$

Die wye steun wat die metaalkarbeenmeganisme vir alkeenmetatese genict het en die eerste ontdekking van metaalkarbyne ${ }^{30.54}$ het daartoe aanleiding gegee dat 'n soortgelyke meganisme as die metaalkarbeenmeganisme vir alkynmetatese voorgestel is, naamlik die metaalkarbynmeganisme wat ook uit twee stappe bestaan:

stap 1 - Skepping van 'n metaalkarbyn langs twee moontlike roetes

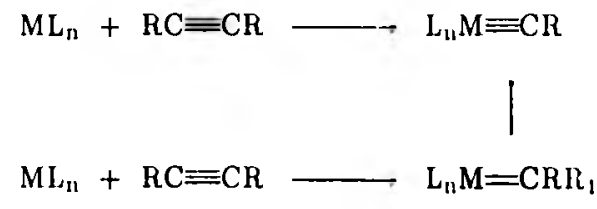

Geen bewyse bestaan egter vir hierdie stap nie en dit is onduidelik hoe 'n Mo(VI)alkilidynkompleks vanaf $\mathrm{Mo}(\mathrm{CO})_{6}$ gevorm kan word. Dit is egter die moeite werd om in verband hiermee te noem dat etilidyntroskomplekse wat Mo(IV) beval, uit 'n reaksic van asynsuur en $\mathrm{MO}(\mathrm{CO})_{6}$ geïsolcer is. ${ }^{55}$

stap 2 - Propagering van die reaksie deur in metaalkarbyn-alkynuitruiling langs 'n metallasiklobutadieentussenverbinding

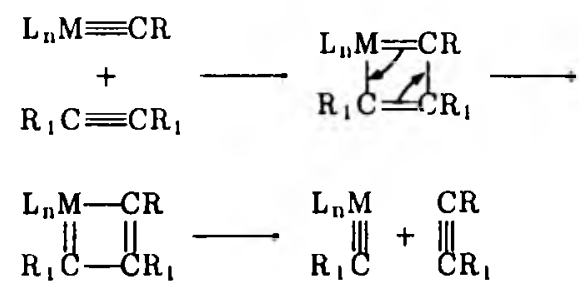

Verskeie bewyse bestaan vir stap 2:

(a) 'n Groot verskeidenheid wolfram- en molibdeenkarbyne van die Schrock-tipe is aktiewe katalisators vir alkynmetatese. ${ }^{33+40}$

(b) 'n Uitgeruilde karbyn is geïsoleer. ${ }^{35}$

(c) In verskeie reaksies is metallasiklobutadieenverbindings geisoleer en die struktuur bepaal. 40.56 .57

Die rol van die fenoliese kokatalisator het ook heelwat aandag gekry en die volgende teorieë is gepostuleer:

(1) Die fenolverbinding tree as voorloper op om die koördinatief onversadigde aktiewe katalisator, ("Mo(CO) ${ }_{3}$ "), te lewer: $7 .+2.43$

$$
\begin{aligned}
& \mathrm{Mo}(\mathrm{CO})_{6}+\mathrm{PhOH} \longrightarrow \\
& {\left[\mathrm{Mo}(\mathrm{CO})_{3}(\mathrm{PhOH})\right] \longrightarrow \mathrm{Mo}(\mathrm{CO})_{3} "}
\end{aligned}
$$

Die groot oormat fenol is nodig om 'n beduidende konsentrasie $\left[\mathrm{Mo}(\mathrm{CO})_{3}(\mathrm{PhOH})\right]$ te verseker eerder as om behulpsaam te wees met die verwydering van die alkyn uit die aanvanklike molibdeenkompleks. Daar is gevind dat die reaksic onreaktief in tolueen is en dat die katalisator eers weer met fenol geregenereer moet word:28 


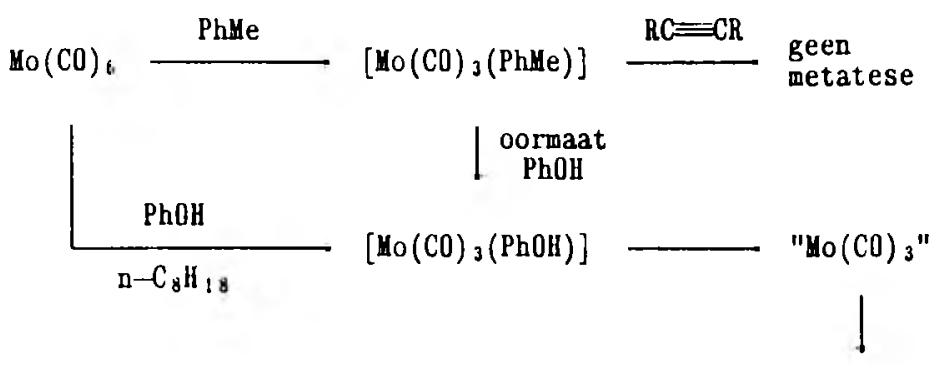

metatese

Aangesien daar ook molibdeendikarbonielkomplekse bekend is wat alkynmetatese kataliseer, is dit moontlik dat die "Mo(CO) 3 "-spesie op die maksimum COinhoud van die aktiewe katalisator dui. ${ }^{7}$

(2) Die rol van die fenolverbinding is om die trippelbindingkarakter van die alkyn deur intermolekulêre waterstofbınding te verswak sodat die alkyn olefinies van aard is, en om isomerisasie van die metallasiklobutadieentussenverbinding te induseer. ${ }^{27}$

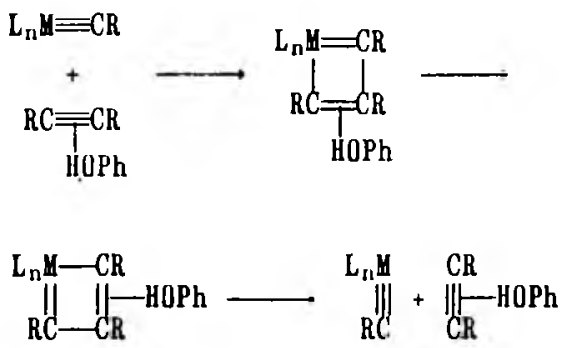

'n Onlangse publikasie ${ }^{5 x}$ toon dat die fenol veroorsaak dat die alkynmetatesereaksie langs 'n karbeenmeganisme kan plaasvind deurdat die alkyn in so ' $n$ mate gepolariseer word dat in metallasiklobutaankation gevorm word:

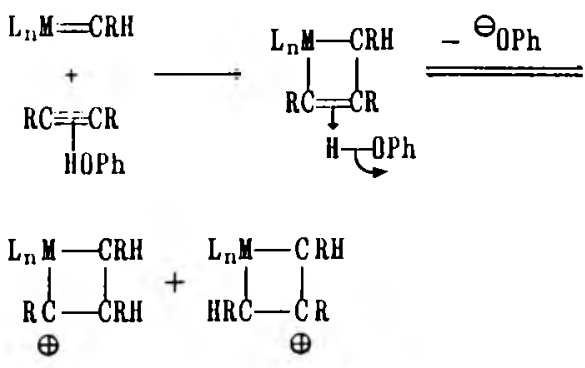

Dit is duidelik dat daar nog nie sekerheid oor die meganistiese verloop en presiese aard van die alkynmetatesereaksic is nie.

\section{I.ITERATUL RVERIYYSINGS}

I. Sclineider. V \& Frolich. P.K. (1931). Formation of Aromatics from lower paraltion. Ind. Eng. Chem. 23. 1405.

2. Banks. R.L. \& Bailey. G.C. (1964). Oletin Disproportionation. Ind. Fing. Chem. Prod. Re's. Deis. 3, 170 .

3. Nin. K.J. (1983). Olciin Me'tahlesis (Aciadenic Press. London).

4. Voston. H.C.M. \& Du Plensis. J.A.K. (1988). 'n Oorsig van Megamismes van dic Oletienmelattesereaksie. S. Affi: Tidski: Natumr11' $\%$. Tegn. 7. 154

5. Reme- J.J. \& Stewart. A. (1977). Olelin Metathesis. Catalsisis, 1. 277.

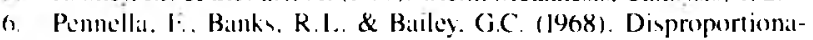
(ion of Alkines. (Hem. Commmm. 1548,

7. Mortreur. A. \& Blanchiard. M. (1974). Metaltesis of Alkynes by a Molybdenum Hexacarbonyl-Resorcinol Catalyst. J. Chem. Soc., Chem. Commmm., 786.

8. Morlreux. A., Petit. F. \& Blanchard. M. (1978). ${ }^{13} \mathrm{C}$ Tracer Studies of Alkyne Metathesis. Tetrahedron Lett., 4967

9. Mortreux, A., Petit, F. \& Blanchard, M. (1980). Improving Selectivity and ${ }^{13} \mathrm{C}$ Experiments during Metathesis of Alkynes on $\mathrm{MoO}_{3}-\mathrm{SiO}$, Catalysts, J. Mol. Catal. , 8. 97.

10. Mortreux, A. \& Blanchard, M. (1972). Disproportion des Hydrocarbures Acélyléniques sur un Catalyseur $\mathrm{MoO}_{3}-\mathrm{SiO}_{2}, \mathrm{Bull}$. Soc: Chim. Fr. 1641

I1. Moulijn, J. A., Reitsma, H.J. \& Boelhouwer, C. (1972). Disproportionation and Cyclotrimerization of Alkynes Over Supported Tungsten Oxide, J. Catal., 25, 434.

12. (a) Farona, M.F., Lofgren, P.A. \& Woon. P.S. (1974). Polymerization of Phenylacetylene Catalysed by Arene-Group VIB Tricarbonyls, J. Chem. Soc., Chem. Commun. 246. (b) Woon, P.S. \& Farona, M.F. (1974). Polymerization of Acetylenes Catalyzed by Arene-Group VIB Tricarbonyls, J. Polym, Sci., Polym. Chem. Eal., 12, 1749.

13. Filippo. J.S., Sowinski, A.F. \& Romano, L.J. (1975). Chlorination of Alkenes and Alkynes with Molybdenum(V)chloride, J. Am. Chem. Soc. , 97, 1599

14. Katz. T.J. \& Sivavec, T.M. (1985). Metal-Catalyzed Rearrangemen of Alkene-Alkynes and the Stereochemistry of Metallacyclobutene Ring Opening. J. Am. Chem. Soc., 107. 737.

15. Kolshorn, H., Meier, H. \& Müller, E. (1972). Photochemie in Gegewenwart van Metallcarbonylen II. Die Thermische und Photochemische Reaktion von Cyclooctin und Molybdänhexacarbonyl. Tetrahedron Lett., 1589.

16. (a) White, J.F. \& Farona, M.F. (1971). Homogeneous Catalysis of Friedel-Crafts-Type Reactions by Arene-Group VIB Tricarbonyls. J. Am. Chem. Soc., 93, 2826. (b) White, J.F. \& Farona, M.F. (1973). Friedel-Crafts Reactions Catalyzed by Arenetricarbonylmolybdenum. J. Orgamomet. Chem., 63, 329. (c) Tsonis, C.P. \& Farona, M.F. (1976) Friedel-Crafts Reactions Catalyzed by Polystyrenetricarbonylmolybdenum, J. Orgemomet, Chem., 114. 293.

17. Dear. R.E.A. \& Pattison, F.L.M. (1963). Toxic Fluorine Compounds XVIII. The Synthesis of the Toxic Principle of Dichaperalum Tox icanmm (18-Fluoro-cis-9-ocladecenoic acid) and Related $\omega$-Fluoro Unsaturated Acids, J. Am. Chem. Soc, 85, 622.

18. Petit, M. . Mortreux, A. \& Petit, F. (1982). Homogeneous Metathesis of Functionalized Alkynes, J. Chem. Soc., Chem. Commum., 1385.

19. Lindlar. H. (1952). Ein Neuere Katalysator Für Selektive Hydrierungen, Hels: Chim. Acka., 35, 446.

20. Levisalles, J. \& Villemin, D. (1980). Metathese D'Acetates DAAlcohols $\omega$-Insalures. Synthese de Pheromones D'Insectes, Tetrahedron, 36, 3181 .

21. Chaloner. Penny A. (1986). Handbook of Coordination Catalysis in Organic Chemistry (Butterworth, London). p. 948-951.

22. Greco, A.. Pirinoli, F. \& Dall'Asti, G. (1973). Reactions of Molybdenum and Tungsten Halides with Acetylenic Hydrocarbons: An Approach to the Structure and Pathways of Formation of Metathesis Catalysts. J. Organomet, Chem., 60, 115.

23. Schrock, R.R., Weinstock, I.A., Horton, A.D., Liu, A.H. \& Schofield. M.H. (1988). Preparation of Rhenium(VI) Monoimido Alkylidyne Complexes and Metathesis of Acetylenes via Rhenacyclobuladiene Intermediates, J. Am. Chem. Soc., 110. 2686.

24. Howe. R.F., Davidson, D.E. \& Whan. D. A. (1972). Infraed Spectra and Catalytic Activity of Supported Molybdenum Hexacarbonyl, $J$. Chem. Soc., Faraday Trans. 1, 68, 2266

25. Ermakov, Yu I. (1976). Supported Catalysts obtained by Interaction of Organometallic Compounds of Transition Elements with Oxide Supports, Catal. Rei: - Sci. Eng., 13. 77.

26. Naccache, C., Bandicra, J. \& Dufaux. M. (1972). An Electron Spin Resonance Sludy of Cation Radicals on the Surface of $\mathrm{MoO}_{3}-\mathrm{SiO}_{2}$ Catalysts, J. Catal., 25, 334.

27. Bencheick, A., Petit, M. . Mortreux, A.\& Petit, F. (1982). New Active and Selective Catalysts for Homogeneous Metathesis of Disubstituted Alkynes, J. Mol. Catal., 15, 93 .

28. Devarajan, S., Walton, D.R.M. \& Leigh. G.J. (1979). Homogene- 
ous Acetylene Metathesis. Nature of the Catalytically Active Species in the $\left[\mathrm{Mo}(\mathrm{CO})_{6}\right] /$ Phenol System, J. Orgamomet. Chem., 181 , 99.

29. Villemin. D. en Cadiot, P. (1982). Homogeneous Metathesis of Functionalised Acetylenes. Tetrahedron Lett., 23, 5139.

30. Fischer, E.O. Kreis, G., Kreiter C.G.. Mueller, J., Huttner, G. \& Lorenz. H. (1973). Transition Metal Carbene Complexes. 60. transHalo|alkyl(aryl)earbyneltetracarbonyl Complexes of Chromium, Molybdenum, and Tungsien. New Complex Type with Transition Metal-Carbon Triple Bond, Angew: Chem., Im. Ed. Engl, 12, 564.

31. (a) Clark. D.N. en Schrock, R.R. (1978). Multiple Metal-Carbon Bonds. 12. Tungsten and Molybdenum Neopentylidyne and some Tungsten Neopentylidyne Complexes. J. Am. Chem. Soc, 100, 6774. (b) Schrock. R. R., DePue, R.T. Feldman, J.. Schaverien, C.J., Dewan. J.C. \& Liu. A.H. (1988). Preparation and Reactivity of Several Alkylidene Complexes of the type $\mathrm{W}\left(\mathrm{CHR} \mathrm{R}^{1}\right)\left(\mathrm{N}-2.6-\mathrm{C}_{6} \mathrm{H}_{3}\right.$-i$\left.\mathrm{Pr}_{2}\right)(\mathrm{OR})_{2}$ and Related Tungstacyclobutane Comlexes. Controlling Metathesis Activity through the Choice of Alkoxide Ligand, J. Am. Chem. Soc., 110, 1423. (c) Schrock, R.R. (1986). High-OxidationState Molybdenum and Tungsten Alkylidyne Complexes, Acc. Chem. Re's., 19. 342.

32. Kat7, T.J. \& McGinnis, J. (1975). The Mechanism of the Olefin Metathesis Reaction, J. Am. Chem. Soc., 97. 1592.

33. Weiss. K. (1988). In Carbyne Complexes, Fischer. H.. Hofimann. P., Kreissl. F.R., Schrock, R.R., Schubert, U. \& Weiss. K. (VCH Verlagsgesellschaft. Weinheim) p. 205.

34. Churchill. M.R. Z Ziller, J.W. Freudenberger. J.H. \& Schrock, R.R. (1984). Metathesis of Acetylenes by Triphenyltungstenacyclobutadiene Complexes and the Crystal Structure of $\mathrm{W}\left(\mathrm{C}_{3} \mathrm{E}_{3}\right) \mid \mathrm{O}-2,6-\mathrm{C}_{6} \mathrm{H}_{3}(\mathrm{i}-$ $\operatorname{Pr})_{2} l_{3}$. Organometallics, 3, 1554.

35. Wengrovious, J.H., Sancho, J. en Schrock, R.R. (1981). Metathesis of Acetylenes by Tungsten (VI)-Alkylidyne Complexes, J. Am. Chem. Soc., 103, 3932.

36. Leigh, G.J. Rahman. M.T. en Walton. D. R.M. (1982). Carbon-Carbon Triple-bond Fission in the Homogeneous Catalysis of Acetylene Metathesis, J. Chem. Soc, Chem. Commun., 541.

37. McCullough, L.G., Schrock, R.R., Dewan, J.C. \& Murdzek. J.C. (1985) Preparation of Trialkoxymolybdenum(VI) Alkylidyne Complexes, Their Reactions with Acetylenes, and the X-ray Structure of $\left.\left.\mathrm{Mo}_{3} \mathrm{C}_{3}\left(\mathrm{CMe}_{3}\right)_{2}\right] l \mathrm{OCH}\left(\mathrm{CF}_{3}\right)_{2}\right]_{2}\left(\mathrm{C}_{5} \mathrm{H}_{5}\right)_{2}$, J. Am. Chem. Soc., 107. 5987.

38. Freudenberger, J.H., Schrock, R.R., Churchill, M.R., Rheingold, A.L. \& Ziller. J.W. (1984). Metathesis of Acetylenes by (Fluoroalkoxy) Iunstenacyclobutadiene Complexes and the Crystal Structure of $\mathrm{W}\left(\mathrm{C}_{3} \mathrm{Et}_{3}\right)\left[\mathrm{OCH}\left(\mathrm{CF}_{3}\right)_{2}\right]_{3}$. A Higher Order Mechanism for Acetylene Metathesis, Orgamometallics, 3, 1563.

39. Schrock, R.R.. Freudenberger, J.H., Listemann, M.L. en McCullough, L.G. (1985). Recent Advances in the Chemistry of Well-defined Olefin and Acetylene Metathesis Catylysts. J. Mol. Catal., 28. I.
40. McCullough, L.G. \& Schrock, R.R. (1984). Metathesis of Acetylenes by Molybdenum(VI) Alkilidyne Complexes, J. Am. Chem. Soc: 106. 4067.

41. Siockel. E. (1978). Investigations of Selected Catalysts for Oletin Metathesis, Diss. Abstr. Int. B, 38, 5948.

42. Mortreux, A., Dy. N. \& Blanchard, M. (1975/76). Metathesis of Acetylenic Hydrocarbons on Molybdenum-based Catalysts, J. Mol Catal, 1. 101.

43. Mortreux, A., Delgrange, J.C.. Blanchard. M. \& Lubochinsky. B. (1977). Role of Phenol in the Metathesis of Acetylenic Hydrocarbons on Catalysts based on Molybdenum Hexacarbonyl, J. Mol. Caral., 2, 73 .

44. Sancho, J. \& Schrock, R.R. (1982). Acetylene Metathesis by Tungsien(VI) Alkylidyne Complexes, J. Mol. Catal. 15. 75

45. Bowden. F.L. \& Lever, A.B.P. (1968). The Transition Metal Chemistry of Acetylenes, Orgamomet. Chem. Re's, 3. 227.

46. Katz. T.J. en Lee, S.J. (1980). Initiation of Acetylene Polymerization by Metal Carbenes, J. Am. Chem. Soc., 102, 422.

47. Hughes, W. B. (1977). Application of Olefin Metathesis to Organic Synthesis, Am. N. Y. Acad. Sci., 295, 271

48. Höcker. H. en Musch, R. (1975). Oligomerization of Cyclodecyne in the Presence of a Metathesis-Catalyst, Makromol. Chem., 176, 3117

49. Grubbs, R.H. (1982). Alkene and Alkyne Metathesis Reactions. In Comprehensive Organometallic Chemistry; Wilkinson, G. ed. (Pergamon Press, Oxford), 8, p. 500.

50. Whitesides, G.M. \& Ehmann. W.J. (1969). The Cyclotrimerization of 2-Butyne-1,I.1-d $\mathrm{d}_{3}$ by Transition Metal Catalysts, J. Am. Chem. Soc., 91, 3800.

51. Efraty, A. (1977). Cyclobutadienemetal Complexes, Chem. Ren., 77, 691

52. Fritch, J.R. en Volhardt, K.P.C. (1979). Cyclobutadiene-Metal Complexes as Potential Intermediates in Alkyne Metathesis: Flash Vacuun Pyrolysis of Substituted $\eta^{4}$-Cyclobuladiene- $\eta^{5}$-cyclopentadienylcobalt Complexes, Angew: Chem., Im. Ed. Engl., 18, 409.

53. Nesmeyanov, A.N. (1972). My Way in Organometallic Chemistry Ads: Organomet, Chem., 10. 56.

54. Fischer E.O. (1976). On the Way to Carbene and Carbyne Complexes. Ad: Organomet, Chem., 14, 1 .

55. Bino. A., Cotton, F.A., Dori, Z. \& Kolthammer, B.W.S. (198I) Trinuclear Mo-Mo-Bonded Cluster Cation with One Oxygen Atom Cap and One Ethylidyne Cap. J. Am. Chem. Soc., 103, 5779.

56. Pederson, S.F., Schrock, R.R. Churchill, M.R.\& Wasserman, H.J. (1982). Reaction of Tungsten(VI) Alkylidyne Complexes with Acetylenes to give Tungstenacyclobutadiene and Tungsten Cyclopentadienyl Complexes, J. Am. Chem. Soc, 104,6808.

57. Bursten, B.E. (1983). On the Stability of Early-Transition-Metal metallacyclobutadienc Complexes, J. Am. Chem. Soc. 105. 121.

58. Bages, S., Petit, M., Mortreux, A. \& Petit, F. (1990). Etude de la Métathèse des Hydrocarbures Acetyleniques Catalysée par le Système 'O,Mo(acac), $-\mathrm{AlEt}_{3}-\mathrm{PhOH}$, J. Mol. Catal., 59, L25. 